\title{
Blood ammonia and glutamine as predictors of hyperammonemic crises in patients with urea cycle disorder
}

\author{
Brendan Lee, MD, PhD ${ }^{1,2}$, George A. Diaz, MD³, William Rhead, MD, PhD ${ }^{4}$, Uta Lichter-Konecki, MD5,

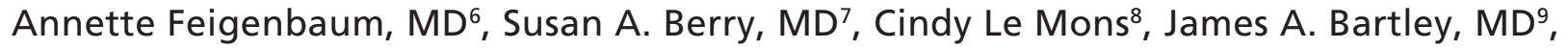 \\ Nicola Longo, MD, $\mathrm{PhD}^{10}$, Sandesh C. Nagamani, MD1, William Berquist, MD ${ }^{11}$, \\ Renata Gallagher, $\mathrm{MD}, \mathrm{PhD}^{12}$, Dennis Bartholomew, $\mathrm{MD}^{13}$, Cary O. Harding, $\mathrm{MD}^{14}$, \\ Mark S. Korson, MD ${ }^{15}$, Shawn E. McCandless, MD ${ }^{16}$, Wendy Smith, MD ${ }^{17}$, Stephen Cederbaum, MD ${ }^{18}$, \\ Derek Wong, MD ${ }^{18}$, J. Lawrence Merritt II, MD ${ }^{19}$, Andreas Schulze, MD, PhD, \\ Gerard Vockley, MD, PhD ${ }^{20}$, David Kronn, MD21, Roberto Zori, MD22, Marshall Summar, MD ${ }^{5}$, \\ Douglas A. Milikien, MS'23, Miguel Marino, PhD ${ }^{15}$, Dion F. Coakley, Pharm D24, \\ Masoud Mokhtarani, MD24, the UCD Consortium and Bruce F. Scharschmidt, MD24
}

Purpose: The aim of this study was to examine predictors of ammonia exposure and hyperammonemic crises in patients with urea cycle disorders.

Methods: The relationships between fasting ammonia, daily ammonia exposure, and hyperammonemic crises were analyzed in $>100$ patients with urea cycle disorders.

Results: Fasting ammonia correlated strongly with daily ammonia exposure $(r=0.764 ; P<0.001)$. For patients with fasting ammonia concentrations $<0.5$ upper limit of normal (ULN), 0.5 to $<1.0$ ULN, and $\geq 1.0 \mathrm{ULN}$, the probability of a normal average daily ammonia value was 87,60 , and $39 \%$, respectively, and $10.3,14.1$, and $37.0 \%$ of these patients, respectively, experienced $\geq 1$ hyperammonemic crisis over 12 months. Time to first hyperammonemic crisis was shorter $(P=0.008)$ and relative risk $(4.5 \times ; P=0.011)$ and rate $(\sim 5 \times, P=0.006)$ of hyperammonemic crises were higher in patients with fasting ammonia $\geq 1.0$ ULN vs. $<0.5 \mathrm{ULN}$; relative risk was even greater $(20 \times ; P=0.009)$ in patients $\geq 6$ years old. A 10 - or $25-\mu \mathrm{mol} / \mathrm{l}$ increase in ammonia exposure increased the relative risk of a hyperammonemic crisis by 50 and $>200 \%(P<0.0001)$, respectively. The relationship between ammonia and hyperammonemic crisis risk seemed to be independent of treatment, age, urea cycle disorder subtype, dietary protein intake, or blood urea nitrogen. Fasting glutamine correlated weakly with daily ammonia exposure assessed as 24-hour area under the curve and was not a significant predictor of hyperammonemic crisis.

Conclusion: Fasting ammonia correlates strongly and positively with daily ammonia exposure and with the risk and rate of hyperammonemic crises, suggesting that patients with urea cycle disorder may benefit from tight ammonia control.

Genet Med advance online publication 11 December 2014

Key Words: glycerol phenylbutyrate; hyperammonemia; RAVICTI; sodium phenylbutyrate

\section{INTRODUCTION}

Urea cycle disorders (UCDs) are inborn errors of metabolism involving deficiencies of enzymes required for ureagenesis. They are manifested as acute and chronic hyperammonemia, and medical management is aimed at reducing ammonia concentrations through the restriction of protein intake and the use of alternative-pathway drugs to enhance excretion of waste nitrogen. Current UCD treatment guidelines indicate that ammonia should be kept within normal limits but otherwise provide limited guidance on the specific timing of blood draws or target levels. ${ }^{1}$

Glycerol phenylbutyrate (GPB; HPN-100, RAVICTI; Hyperion Therapeutics, Brisbane, CA) is a sodium- and sugarfree ammonia-lowering agent that was approved in the United States in 2013 for UCD patients $\geq 2$ years of age who cannot be managed by dietary protein restriction and/or amino acid supplementation alone. The GPB clinical trials compared 24-hour ammonia concentrations during short-term steady-state dosing

\footnotetext{
${ }^{1}$ Department of Molecular and Human Genetics, Baylor College of Medicine, Houston, Texas, USA; ${ }^{2}$ Howard Hughes Medical Institute, Chevy Chase, Maryland, USA; ${ }^{3}$ Icahn School of Medicine at Mount Sinai, New York, New York, USA; ${ }^{4}$ The Medical College of Wisconsin, Milwaukee, Wisconsin, USA; ${ }^{5} \mathrm{Children's} \mathrm{National} \mathrm{Medical} \mathrm{Center,} \mathrm{Washington,} \mathrm{DC,}$ USA; ${ }^{6}$ The Hospital for Sick Children, University of Toronto, Toronto, Ontario, Canada; ${ }^{7}$ University of Minnesota, Minneapolis, Minnesota, USA; ${ }^{8}$ National Urea Cycle Disorders Foundation, Pasadena, California, USA; ${ }^{9}$ Miller Children's Hospital, Long Beach, California, USA; ${ }^{10}$ University of Utah, Salt Lake City, Utah, USA; ${ }^{11}$ Stanford University, Palo Alto, California, USA; ${ }^{12}$ Children's Hospital Colorado, Aurora, Colorado, USA; ${ }^{13}$ Nationwide Children's Hospital, Columbus, Ohio, USA; ${ }^{14}$ Oregon Health \& Science University, Portland, Oregon, USA; ${ }^{15}$ Tufts Medical Center, Boston, Massachusetts, USA; ${ }^{16}$ University Hospitals Case Medical Center and Case Western Reserve University, Cleveland, Ohio, USA; ${ }^{17}$ Maine Medical Center, Portland, Maine, USA; ${ }^{18}$ University of California, Los Angeles, Los Angeles, California, USA; ${ }^{19}$ University of Washington, Seattle, Washington, USA; ${ }^{20}$ University of Pittsburgh, Pittsburgh, Pennsylvania, USA; ${ }^{21}$ Westchester Medical Center, Westchester, New York, USA; ${ }^{22}$ University of Florida, Gainesville, Florida, USA; ${ }^{23}$ Accudata Solutions, Lafayette, California, USA; ${ }^{24}$ Hyperion Therapeutics, Brisbane, California, USA. Correspondence: Brendan Lee (blee@bcm.edu)
} 
with GPB or sodium phenylbutyrate (NaPBA) among $80 \mathrm{UCD}$ patients aged 2 months to $>70$ years and studied longer-term outcomes, including hyperammonemic crises (HACs), among 100 UCD patients, including 49 pediatric patients, treated with GPB for up to 1 year.

This report takes advantage of $>1,000$ prospectively collected timed blood samples to analyze correlates of daily ammonia exposure, assessed as 24-hour area under the curve, as well as the utility of ammonia and glutamine as predictors of HACs.

\section{MATERIALS AND METHODS}

\section{Clinical studies}

Data from four short-term switchover (SO) studies and three 12-month safety extension studies of adult and pediatric UCD patients were included in the analyses; all have been previously described. Protocols UP1204-003, HPN-100005SO, and HPN-100-012SO were short-term, open-label, fixed-sequence SO studies that evaluated ammonia control during equivalent dosing of GPB versus NaPBA. ${ }^{2-4}$ Study HPN-100-006 was a pivotal, randomized, double-blind, active-controlled, crossover study that established the noninferiority of GPB to NaPBA as assessed by venous ammonia. ${ }^{5}$ Most patients completing these protocols plus additional patients (a total of 100 patients, of whom 49 were pediatric) were enrolled into safety extension studies and received GPB for 12 months. ${ }^{5-7}$

All protocols and informed consents were reviewed and approved by the investigational review board of each participating institution before initiation. Eligible subjects had a confirmed or clinically suspected UCD and had been receiving NaPBA before enrollment. In all studies patients received GPB three times daily (or more frequently in small children to match their prior NaPBA schedule) at a daily dose equivalent to their previously prescribed NaPBA dose.

All procedures were in accordance with the ethical standards of the responsible committee on human experimentation (institutional and national) and with the Helsinki Declaration of 1975, as revised in 2000. Informed consent was obtained from all patients.

The ClinicalTrials.gov identifiers for the studies were NCT00551200, NCT00947544, NCT00947297, NCT00992459, and NCT01347073.

\section{Ammonia and glutamine measurements}

During the crossover studies, serial venous blood samples for ammonia analyses were collected over 24 hours after the patient had received 1-2 weeks of dosing (steady state) with either NaPBA or GPB. Samples for glutamine and ammonia were collected after overnight ( $\geq 4-6$ hours) fasting and before breakfast and the first daily drug dose; additional ammonia samples were collected at 8,12 , and 24 hours after the dose for pediatric patients and approximately every 4 hours for adults. During the 12-month studies, blood samples were collected monthly for ammonia and glutamine analyses, and information on HACs was recorded.
Baseline ammonia was defined as the screening or month 0 value when the patient was taking NaPBA before receiving GPB. A HAC was defined as compatible clinical symptoms associated with one or more ammonia levels $\geq 100 \mu \mathrm{mol} / \mathrm{l}$. Ammonia and HAC data were also collected retrospectively for up to 12 months before enrollment in each study while patients were receiving NaPBA. Ammonia and glutamine concentrations were measured by an accredited hospital laboratory at each study site, and ammonia values were normalized to a standard range of $9-35 \mu \mathrm{mol} / \mathrm{l}$.

\section{Statistical analyses}

Short-term studies. Fasting ammonia concentrations were determined from the predose and 24-hour time points for steady-state measurements; maximum concentration represented the highest concentration determined during the 24-hour period. Ammonia 24-hour area under the concentration versus time curve from time 0 (predose) to 24 hours $\left(\mathrm{AUC}_{0-24}\right)$ was calculated using the linear trapezoid rule, and average daily ammonia was calculated as $\mathrm{AUC}_{0-24}$ divided by time. The relationship between fasting ammonia and $\mathrm{AUC}_{0-24}$ was analyzed using logistic regression. Potential covariates examined included age, weight, drug type, and total daily dietary protein intake. Prediction intervals were calculated using standard least-squares methods and bootstrap methods. ${ }^{8}$ Bootstrap intervals are less dependent on the observed data than standard intervals and therefore provide a more credible predication interval.

Fasting ammonia values were also divided into categories $(<0.5,0.5$ to $<1.0$, and $\geq 1.0)$ relative to the upper limit of normal (ULN). The probability that a patient whose fasting ammonia falls within a given category would have normal total daily ammonia exposure was modeled using generalized estimating equations assuming an exchangeable correlation structure and a logit link function. Confidence limits around the outcome probabilities were computed using the bootstrap method. ${ }^{8}$

Twelve-month studies. The time to the first HAC was determined by Kaplan-Meier analysis ${ }^{9}$ for the entire population, by age group, dichotomous age group, gender, and baseline ammonia ULN categories. Survival distributions between covariate values were computed using a log-rank test. ${ }^{10} \mathrm{Cox}$ regression models were used to quantify the relative risk of time to first HAC.

The relationship between total ammonia exposure over time and the risk of HAC occurrence was explored by calculating time-normalized AUC for ammonia for patients with and without a HAC during the 12-month treatment with GPB. The AUC for ammonia, measured monthly, was calculated for each patient using the trapezoid rule over the course of the 12-month study. Time-normalized AUC was calculated by dividing AUC by the final study month of an ammonia assessment.

For the cumulative rate of HAC over 12 months, a negative binomial regression was performed with a flexible scale parameter to account for overdispersion of the HAC count data ${ }^{11}$; an 
offset accounted for varying time in the study between patients. A multivariable negative binomial regression of HAC rate was also was developed to adjust for potential confounders.

A linear mixed regression model was used to analyze admission, peak, and discharge ammonia concentrations by patient characteristics. Log-transformed values were used for peak and discharge ammonia concentrations, and the model controlled for clustering of outcomes for patients who had more than one HAC. ${ }^{12}$ A generalized estimating equation logistic regression model was used to assess the association between the duration of the HAC and baseline ammonia values. ${ }^{13}$ Spearman correlations were used to assess the relationship between baseline ammonia and glutamine concentrations.

\section{RESULTS}

The analyses included $>1,000$ ammonia results from 114 patients with UCD, including 100 patients (51 adult, 49 pediatric) in the 12 -month dosing protocols. Except for fasting ammonia, baseline characteristics (age, gender, UCD subtype) were similar across baseline ammonia ULN categories, although there was a slightly higher proportion of patients aged 6-11 years in the 0.5 to $<1.0$ ULN group than in the other two groups, and mean baseline glutamine values were slightly higher in the $\geq 1.0$ ULN group than in the other ULN groups (Table 1).

\section{Short-term studies}

Blood ammonia concentrations fluctuated considerably over 24 hours; fasting ammonia values from morning blood draws exhibited the least variability (Supplementary Figure S1 online). There was a strongly positive and statistically significant relationship between fasting ammonia and $\mathrm{AUC}_{0-24}(r=0.764$; $P<0.0001$ ) (Figure 1). There was no discernable difference in the relationships observed with NaPBA and GPB treatment, so data for the two drugs were combined. None of the potential covariates examined (e.g., age, weight, gender, and dietary protein) had a clinically or statistically significant impact on the model, and these covariates were not included in the final model.

When AUC and maximum daily ammonia concentration outcomes were modeled using ordered ULN categories, patients with a fasting ammonia $<0.5$ ULN had a greater likelihood of having a normal average daily ammonia value than patients with a fasting ammonia in other categories (inset in Figure 1).

Table 1 Patient characteristics in relation to baseline ammonia values

\begin{tabular}{|c|c|c|c|c|}
\hline \multirow[b]{2}{*}{ Variable } & \multicolumn{3}{|c|}{ Baseline ammonia category } & \multirow[b]{2}{*}{ Total $(N=100)$} \\
\hline & $<0.5$ ULN $(n=39)$ & $0.5-<1.0$ ULN $(n=34)$ & $\geq 1.0$ ULN $(n=27)$ & \\
\hline Mean age (SD), years & $22.9(17.7)$ & $17.4(15.0)$ & $18.6(12.8)$ & $19.6(15.9)$ \\
\hline \multicolumn{5}{|l|}{ Age group, $n(\%)$} \\
\hline Adult ( $\geq 18$ years old) & $22(56.4)$ & $14(41.2)$ & $15(55.6)$ & $51(51.0)$ \\
\hline Pediatric (<18 years old) & $17(43.6)$ & $20(58.8)$ & $12(44.4)$ & $49(49.0)$ \\
\hline$<2$ years old & $3(7.7)$ & $2(5.9)$ & $2(7.4)$ & $7(7.0)$ \\
\hline 12-17 years old & $2(5.1)$ & $4(11.8)$ & $3(11.1)$ & $9(9.0)$ \\
\hline \multicolumn{5}{|l|}{ Gender, $n(\%)$} \\
\hline Male & $15(38.5)$ & $9(26.5)$ & $9(33.3)$ & $33(33.0)$ \\
\hline Female & $24(61.5)$ & $25(73.5)$ & $18(66.7)$ & $67(67.0)$ \\
\hline \multicolumn{5}{|c|}{ Fasting laboratory values, mean (SD) } \\
\hline \multicolumn{5}{|l|}{ UCD subtype, $n(\%)$} \\
\hline ARG & $1(2.6)$ & $0(0.0)$ & $1(3.7)$ & $2(2.0)$ \\
\hline ASL & $7(18.0)$ & $5(14.7)$ & $1(3.7)$ & $13(13.0)$ \\
\hline ASS & $4(10.3)$ & $3(8.8)$ & $5(18.5)$ & $12(12.0)$ \\
\hline CPS & $1(2.6)$ & $0(0.0)$ & $0(0.0)$ & $1(1.0)$ \\
\hline $\mathrm{HHH}$ & $2(5.1)$ & $1(2.9)$ & $0(0.0)$ & $3(3.0)$ \\
\hline OTC & $24(61.5)$ & $25(73.5)$ & $20(74.1)$ & $69(69.0)$ \\
\hline \multicolumn{5}{|l|}{ 12-month incidence of $\mathrm{HAC}$} \\
\hline Before taking NaPBA & 0.433 & 0.389 & 1.071 & 0.581 \\
\hline
\end{tabular}

ARG, arginase deficiency; ASL, argininosuccinate lyase deficiency; ASS, argininosuccinate synthase deficiency; BUN, blood urea nitrogen; CPS, carbonyl phosphate synthetase deficiency; GPB, glycerol phenylbutyrate; HAC, hyperammonemic crisis; HHH, hyperornithinemia-hyperammonemia-homocitrullinuria syndrome; NaPBA, sodium phenylbutyrate; OTC, ornithine transcarbamylase deficiency; UCD, urea cycle disorder; ULN, upper limit of normal. 


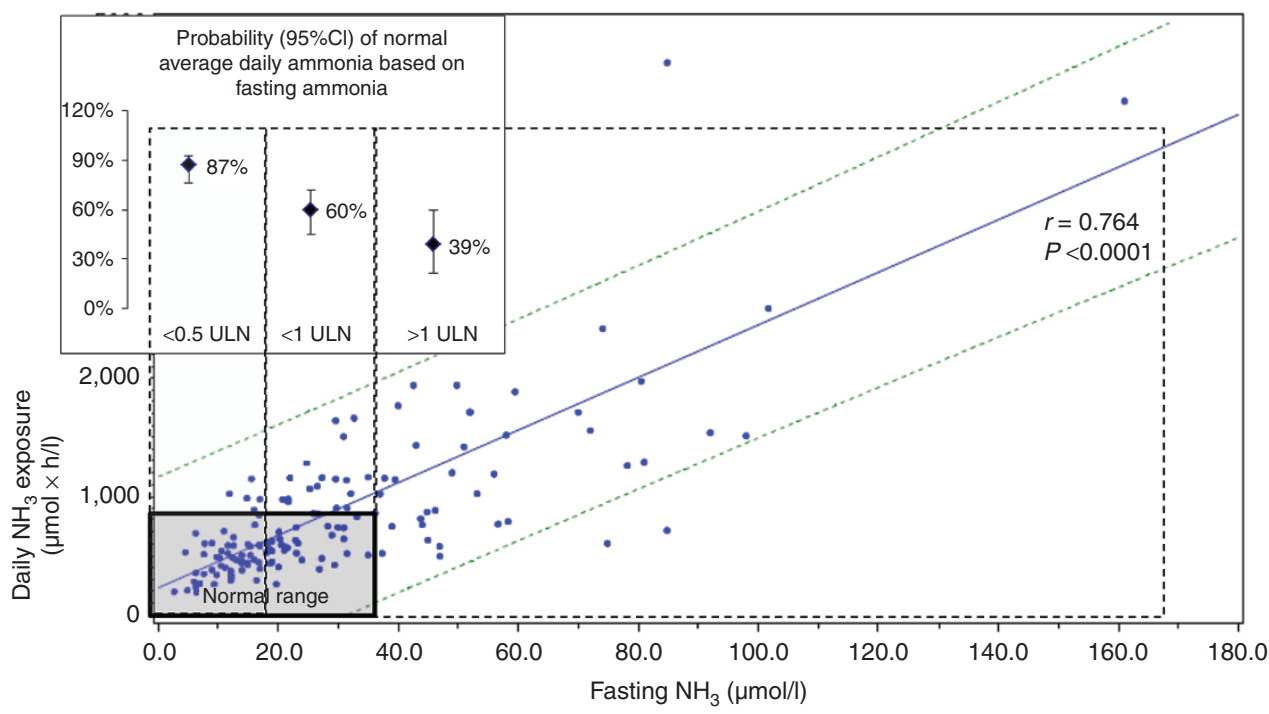

Figure 1 Correlation of fasting ammonia and daily ammonia exposure in short-term crossover studies $(r=0.764 ; P<0.0001 ; N=80)$. Bolded box indicates the normal range. Dashed lines represent $95 \%$ confidence intervals. Dotted boxes indicate fasting ammonia categories. Inset: probability of average daily ammonia being within normal limits by fasting ammonia upper limit of normal categories.

\section{Twelve-month studies}

Ammonia. Ammonia concentrations during up to 12 months of dosing were strongly related to baseline concentrations as categorized in relation to the ULN. Patients whose baseline ammonia was $<0.5$ ULN had mean monthly ammonia concentrations $<35 \mu \mathrm{mol} / \mathrm{l}$ throughout the study, and their ammonia values showed less variability than did those among patients in the higher baseline ammonia categories (Figure 2, top panel). In the ammonia $\geq 1.0$ ULN category, mean fasting ammonia decreased toward the normal range after approximately 3 months of GPB treatment (Figure 2, top panel).

Thirty patients $(30 \%)$ experienced a total of 54 HACs in the 12 months before starting GPB treatment, and 19 patients (19\%) experienced a total of 27 HACs during 12 months of GPB treatment (Table 1). The proportion of patients experiencing a HAC increased in relation to baseline ammonia ULN categories $(P=0.0236)$ and was slightly higher for pediatric than for adult patients. Gender and UCD subtype had no effect on the rate of HACs. Mean (SD) baseline fasting ammonia in patients with a HAC was $41.8(23.7) \mu \mathrm{mol} / \mathrm{l}$ as compared with $25.7(17.8) \mu \mathrm{mol} / \mathrm{l}$ in patients with no HAC $(P=0.0023)$.

The time to first HAC was significantly shorter $(P=0.0077)$ and the risk of HAC higher $(4.5 \times ; P=0.011)$ in patients whose baseline ammonia was $\geq 1.0$ ULN as compared with patients whose baseline ammonia was $<0.5$ ULN (Figure 2). After adjusting for age, gender, and race, patients with one or more baseline ammonia values $\geq 1.0$ ULN had $\sim 5.5$ times the risk of having at least one HAC than patients with baseline ammonia $<0.5$ ULN $(P=0.005)$. When patients $<6$ years old at baseline (who eat more frequently and for whom measurement of fasting ammonia is problematic) were excluded from the analysis, those with baseline ammonia values $\geq 1.0$ ULN had an $\sim 20$ times increase in the risk of having a HAC than patients with baseline ammonia $<0.5$ ULN $(P=0.009)$.
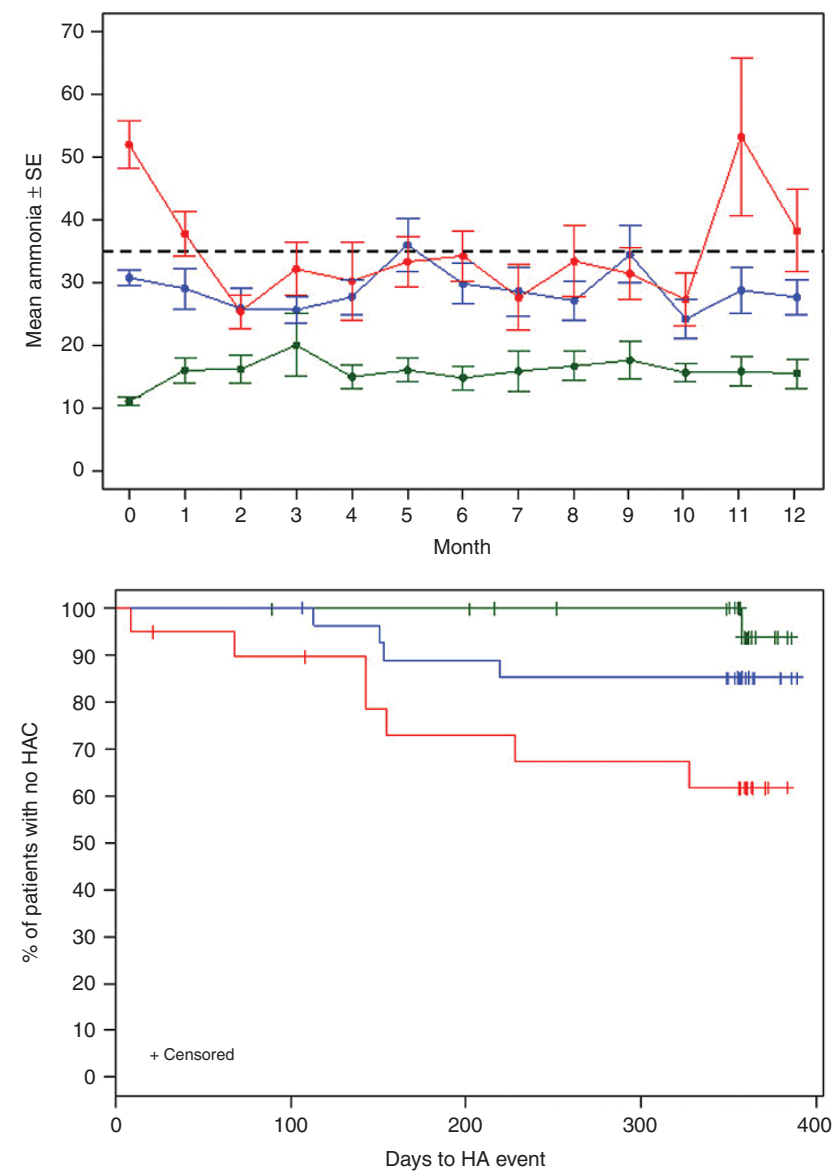

Figure 2 Ammonia concentrations and hyperammonemic crisis (HAC) rate. Top panel: Mean $( \pm \mathrm{SE})$ ammonia values during 12 months of treatment with glycerol phenylbutyrate by baseline fasting ammonia upper limit of normal (ULN) category. Dotted line indicates upper limit of normal range for ammonia $(35 \mu \mathrm{mol} / \mathrm{l})$. Bottom panel: Time to first HAC by baseline ammonia ULN category for patients at least 6 years old at baseline ( $P=0.0077$, log-rank test). Legend for ULN categories: baseline ammonia categories $<0.5$ ULN (green); 0.5 to $<1.0$ (blue), and $>1.0$ ULN (red). 
The median time in the study was 361 days. The overall rate of HACs was 0.288 per patient per year, and the rate was significantly higher among patients with baseline ammonia $\geq 1.0$ ULN than for those with baseline ammonia $<0.5$ ULN ( 0.711 vs. $0.135 /$ patient/year, respectively; $P=0.0057$ ). Total ammonia exposure, calculated as time-normalized AUC during 12 months of GPB dosing, was significantly lower in patients who did not experience a HAC ( 23.6 vs. $35.0 \mu \mathrm{mol} / \mathrm{l} ; P=0.0006$ ). The relative risk of experiencing a HAC increased by about 50 and $270 \%$, respectively, for every 10 and $25 \mu \mathrm{mol} / \mathrm{l}$ increase in ammonia exposure $(P<0.0001)$ (Supplementary Figure $\mathbf{S} 3$ online).

The proportion of patients with baseline ammonia concentrations within each ULN category varied considerably among study sites (Figure 3, top panel), which therefore were grouped into three categories based on the percentage of patients enrolled whose baseline fasting ammonia was $<0.5 \mathrm{ULN}$ at baseline: group 1 (6 study sites; 33 patients, of whom $>50 \%$ had baseline ammonia concentrations $<0.5 \mathrm{ULN}$ ); group 2 ( 3 study sites; 29 patients, of whom $25-50 \%$ had baseline ammonia concentrations $<0.5$ ULN); and group 3 (11 sites; 38 patients, of whom $<25 \%$ had baseline ammonia concentrations $<0.5 \mathrm{ULN}$ ) (Figure 3, bottom panel, and Supplementary Table S1 online). The proportion of patients who experienced a HAC was lowest (12\%) among group 1 sites and increased progressively to 21 and $24 \%$ in the group 2 and 3 sites, respectively (Figure 3, bottom panel). Although the mean age of patients in group 3 was lower as compared with that in group 1 (14.7 vs. 24.5 years), other markers of disease severity, including the proportion of females with ornithine transcarbamylase deficiency and restriction of dietary protein, were similar among the groups.
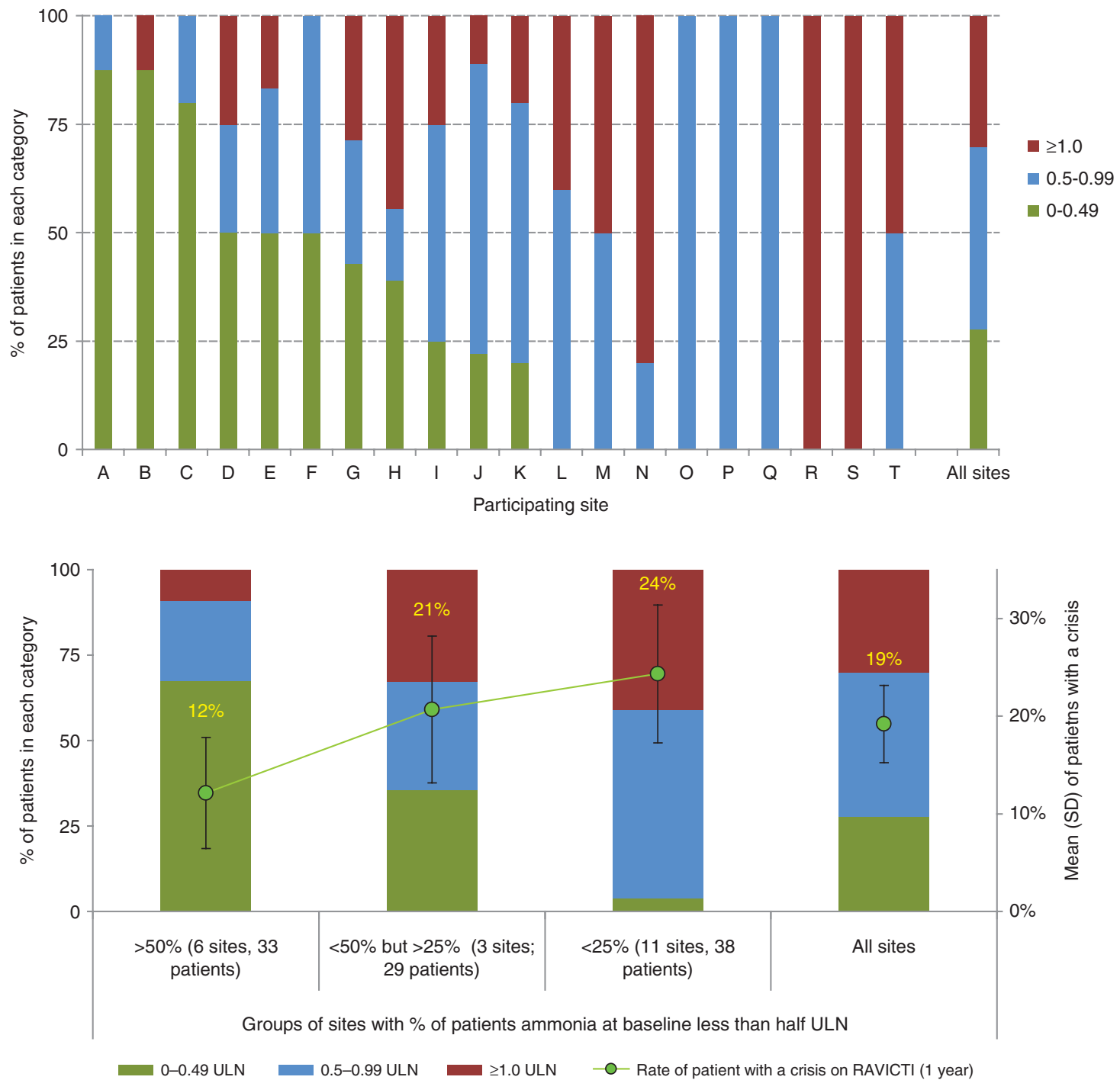

Figure 3 Ammonia concentrations and hyperammonemic crisis rate by study site. Top panel: Percentage of patients at each study site with baseline ammonia $<0.5$ upper limit of normal (ULN), 0.5-1 ULN, and $\geq 1.0$ ULN. Bottom panel: Percentage of patients in each ammonia ULN category by sites, grouped in relation to ammonia concentrations among patients enrolled at the site. Group 1 ( 6 study sites; 33 patients, of whom $>50 \%$ had baseline ammonia concentrations < 0.5 ULN); group 2 ( 3 study sites; 29 patients, of whom 25-50\% had baseline ammonia concentrations <0.5 ULN); and group 3 (11 sites; 38 patients, of whom $<25 \%$ had baseline ammonia concentrations $<0.5 \mathrm{ULN}$ ). 
During the 12-month GPB dosing protocols, HACs were generally of short duration; $48 \%$ resolved within 1 day and $64 \%$ resolved in $<2$ days. Most HACs were triggered by intercurrent illness and infection, and the most commonly reported clinical symptoms reported were vomiting (63\%) and lethargy (37\%), and other (48\%) (Supplementary Table S2 online). Patients with baseline ammonia $<1.0$ ULN tended to have a higher likelihood of experiencing a HAC of $<2$ days' duration than did patients with baseline ammonia $\geq 1.0$ ULN (odds ratio, 3.06), but this difference was not statistically significant.

Glutamine. Monthly glutamine concentrations were generally higher in patients in the higher baseline ammonia ULN groups and, by 3 months of treatment, had decreased in the patients with the highest glutamine concentrations at baseline (Figure 4, top

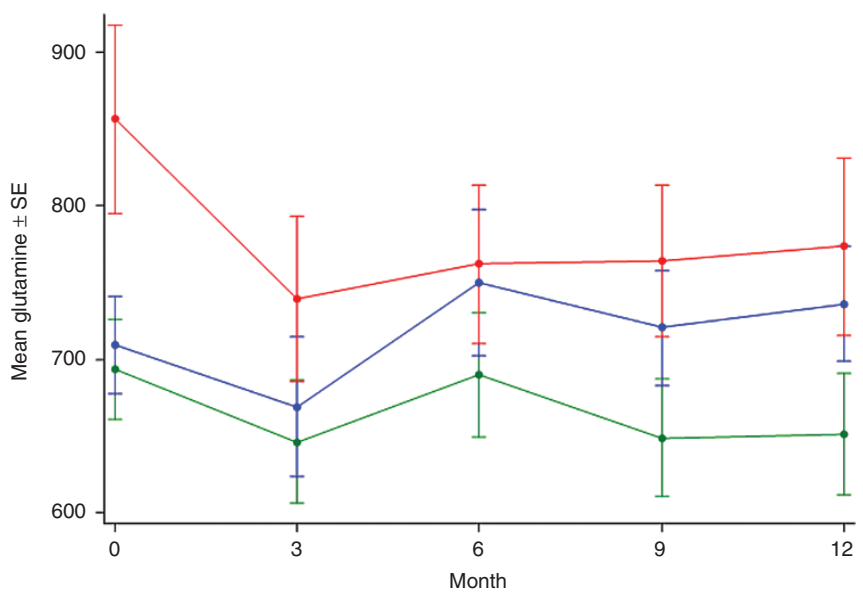

Baseline ammonia ULN $\bullet 0.0-0.49$ ULN $\bullet 0.5-0.99$ ULN $\bullet \geq 1.0$ ULN

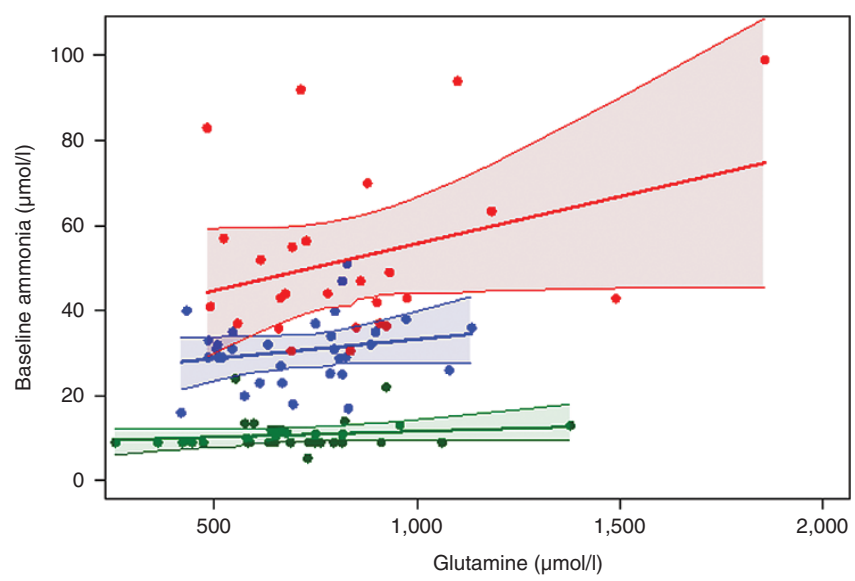

Figure 4 Relationship between glutamine and ammonia concentrations by baseline ammonia categories. Top panel: Glutamine concentrations during 12 months of glycerol phenylbutyrate treatment; mean $( \pm \mathrm{SE})$ monthly glutamine concentrations by baseline ammonia upper limit of normal (ULN) category. Bottom panel: Scatterplot of baseline glutamine by ammonia ULN categories. The overall correlation between glutamine and ammonia was significant $(r=0.27 ; P=0.008)$, whereas none of the correlations within the ammonia ULN categories was significant $(r=0.12,0.18$, and 0.12 , respectively within the three ULN categories; $P=$ nonsignificant for all). panel). However, there was no statistically significant difference between glutamine in patients with or without a $\mathrm{HAC}$ as assessed at baseline ( 782.4 vs. $732.0 \mu \mathrm{mol} / \mathrm{l} ; P=0.150$ ), during the longterm follow-up, or as total glutamine exposure (12-month timenormalized AUC of 720.9 vs. $699.4 \mu \mathrm{mol} / \mathrm{l}^{\star} \mathrm{m} ; P=0.686$ ).

There were statistically significant but comparatively weak correlations between baseline ammonia and glutamine concentrations at the time of enrollment $(r=0.27 ; P=0.008)$, as well as between fasting glutamine concentrations and daily ammonia exposure while taking GPB or NaPBA during the SO studies $(r=0.292, P<0.001)$. However, when the relationship between glutamine and ammonia categories with respect to the ULN was analyzed, glutamine values varied substantially for patients in each category, and the overall correlation seemed to be driven by patients whose baseline ammonia exceeded 1.0 times the ULN (Figure 4). Patients with proximal deficiencies (ornithine transcarbamylase deficiency and carbonyl phosphate synthetase deficiency) showed a stronger correlation between glutamine and ammonia $(r=0.56 ; P=0.020$; Supplementary Figure S2 online). There was no correlation between baseline ammonia and UCD subtype or concentrations of either citrulline $(r=0.04$; $P=0.414)$ or argininosuccinic acid $(r=0.14 ; P=0.158)$.

\section{DISCUSSION}

Twenty-four-hour blood sampling in patients with UCD demonstrated that blood ammonia concentrations fluctuated considerably over 24 hours, even among the patients with comparatively stable and well-controlled UCD who were enrolled in these trials, making interpretation of random blood ammonia concentrations difficult. To identify the optimal timing of blood draws and a target level for ammonia in UCD patients, post hoc statistical analyses of clinical trial data were conducted to assess the short-term relationship between fasting ammonia concentrations and daily ammonia exposure and the long-term relationship between fasting ammonia concentrations and HACs in patients with UCDs.

Analysis of pooled data from the short-term studies showed a strong positive relationship between fasting ammonia and ammonia $\mathrm{AUC}_{0-24}$. Because the wide prediction intervals observed with this analysis compromised the utility of individual levels for predicting daily ammonia exposure, the relationship between fasting ammonia concentrations categorized in relation to the ULN and the likelihood that a patient would have an average or maximal blood ammonia concentration within normal limits was modeled using generalized estimating equations. These analyses demonstrated that fasting ammonia categorized in relation to the ULN ( $<0.5 \mathrm{ULN}, 0.5$ to $<1.0 \mathrm{ULN}$, and $\geq 1.0 \mathrm{ULN}$ ) was strongly predictive of the likelihood that maximal and mean daily ammonia values would be within normal limits. This finding indicates that patients with fasting ammonia concentrations outside the normal range are likely being exposed to large amounts of ammonia during the day, even if they do not exhibit any identifiable symptoms.

To further assess the importance of these correlations, data on ammonia, glutamine, and HACs collected during the 12-month studies were analyzed using the patient groups by baseline ammonia ULN category. The relationship between 
baseline ammonia ULN category and long-term outcome was demonstrated using several approaches. First, monthly ammonia concentrations continued to be lowest in patients with baseline ammonia concentrations $<0.5$ ULN and highest in patients with baseline ammonia $\geq 1.0$ ULN. Second, the proportion of patients experiencing a HAC increased significantly with increasing baseline ammonia ULN category. Third, the time to first HAC was significantly shorter and the risk of HAC was significantly greater in patients with baseline ammonia $\geq 1.0$ ULN than in those with baseline ammonia $<0.5$ ULN. This association between baseline ammonia and HAC risk was even more apparent when patients younger than 6 years of age at baseline (who feed more frequently and for whom true fasting ammonia concentrations are difficult to collect) were excluded from the analysis. Finally, the cumulative rate of HAC was more than five times higher for patients with baseline ammonia $\geq 1$.0 ULN than for patients with baseline ammonia $<0.5$ ULN and was 20 times higher when patients younger than 6 years of age were excluded.

As is true for the relationship between fasting ammonia and daily ammonia exposure, the relationship between fasting ammonia and HAC seems to be independent of treatment. Although fewer crises were experienced by patients during treatment with GPB as compared with the prior year on NaPBA, the relationship between ammonia concentrations and the incidence of HACs was similar before beginning NaPBA treatment and subsequently during treatment with GPB.

The duration of HACs during GPB treatment was generally short, with more than $64 \%$ of crises lasting $<2$ days. Although ammonia values were generally higher in pediatric patients, there were no significant predictors for the admission, peak, or discharge ammonia concentrations during a HAC or for the duration of a HAC.

Compared with ammonia, glutamine seems to be a weaker predictor of HAC. Although glutamine concentrations were higher at baseline in patients who experienced a HAC, there was no significant difference in glutamine between patients who did or did not experience a HAC during the study and no significant association between the rate of HAC and glutamine concentrations during the 12 months of dosing. Glutamine correlated modestly with fasting ammonia at baseline and decreased during GPB treatment in patients with the highest baseline glutamine concentrations. Of interest, the correlation between fasting ammonia and glutamine seemed to be primarily driven by patients with the highest ammonia values, a finding similar to that reported by Maestri et al. ${ }^{14}$ based on multiple samples in a single patient with ornithine transcarbamylase deficiency. In addition, the relationship between ammonia and glutamine seemed to be strongest in patients with the proximal UCD subtypes, ornithine transcarbamylase deficiency and carbonyl phosphate synthetase deficiency (Supplementary Figure S2 online), a finding that corroborates an earlier report by Wilson et al. ${ }^{15}$

Certain limitations of the current analyses deserve emphasis. First, although these timed ammonia and glutamine samples were collected prospectively, the analyses are post hoc. Second, the fact that lower fasting ammonia concentrations correlate strongly with lower average daily ammonia exposure, as well as lower risk and frequency of HAC, could reflect intrinsic differences in disease severity; that is, patients with more severely deficient urea synthesis may exhibit both higher ammonia concentrations and increased susceptibility to crises with even minor triggering events. Two considerations suggest an alternative explanation. First, baseline characteristics including age, gender, and UCD subtype were similar across these ULN groups. Second, data pertaining to ammonia concentrations and the frequency of HAC among patients enrolled at 20 sites in North America, even allowing for possible differences in adherence among patients at different sites, seem to be better explained by different responses to management or differing management approaches rather than regional differences in the severity of illness. In any case, the findings should be interpreted with caution pending further study and prospective validation.

The findings suggest that fasting ammonia is a reliable surrogate for assessing adequacy of disease control; it correlates strongly with total daily ammonia exposure as well as HACs. They further suggest that even mildly elevated ammonia over long periods of time may put patients at risk of HAC and that patients with UCD, especially those $\geq 6$ years old, may benefit from maintaining fasting ammonia concentrations $<0.5$ ULN. More frequent monitoring of fasting ammonia concentrations may require identifying certified clinical laboratories conveniently located for patients and/or further development of portable testing technology analogous to that in use for diabetes.

\section{SUPPLEMENTARY MATERIAL}

Supplementary material is linked to the online version of the paper at http://www.nature.com/gim

\section{ACKNOWLEDGMENTS}

The authors gratefully acknowledge Richard Rowell (Hyperion) for his statistical review and thank the efforts of the study coordinators who made this study possible, including N. Schrager (Icahn School of Medicine at Mount Sinai); A. Donovan, J. Crawford, Pediatric TRU Staff; K. Defouw, J. Balliet (The Medical College of Wisconsin); M. Keuth, N. O'Donnell (Long Beach Memorial Hospital); M. Hussain, E. Bailey, A. Orton, M. Ambreen (The Hospital for Sick Children, University of Toronto, ON, Canada); C. Bailey, M.J. Dunkley (University of Utah); J. Perry, V. de Leon, A. Niemi, K. Cusmano (Stanford University); T. Carlson, J. Parker (University of Minnesota); S. Burr (Children's Hospital Colorado); K. Simpson (Children's National Medical Center); K. Regis (Nationwide Children's Hospital); A. Behrend, T. Marrone (Oregon Health \& Science University); N. Dorrani (University of California, Los Angeles); C. Heggie (Case Western Reserve University); S. Mortenson (Maine Medical Center); S. Deward (Children's Hospital of Pittsburgh); K. Bart, C. Duggan (SNBL); K. Murray, C. Dedomenico (Tufts Medical Center); C. Gross (University of Florida); L. Brody (Seattle Children's Hospital); M. Mullins, S. Carter, A. Tran, J. Stuff, Texas Children's Hospital General Clinical Research Center nursing staff (Baylor College of Medicine); Kathy Lisam (Hyperion); and UCD Consortium members, including Mark L. Batshaw, Men- 
del Tuchman, Matthias R. Baumgartner, Georg Hoffmann, Douglas S. Kerr, Margretta R. Seashore, Tamar Stricker, Susan Waisbren, and Mark Yudoff. The authors also acknowledge the Clinical and Translational Science Awards/General Clinical Research Center grants (Baylor College of Medicine, M01RR00188; Case Western Reserve University, NIDDK 1K08DK074573; Clinical and Translational Science Institute at the Children's National Medical Center, National Institutes of Health (NIH)/National Center for Research Resources, UL1RR31988; Medical College of Wisconsin, UL1RR31973; Mount Sinai School of Medicine, UL1RR29887; Oregon Health \& Science University, UL1RR24140; Stanford University, UL1RR25744; Tufts University, UL1RR25752; University of California, Los Angeles, UL1RR33176; University of Colorado, UL1RR25780; University of Florida, UL1RR29890; University of Minnesota, UL1RR33183; University of Pittsburgh, NIH, UL1TR000005; University of Utah, UL1RR25764; University of Washington, UL1TR000423), the Urea Cycle Disorders Consortium (NIH grant U54HD061221) and grants from the O'Malley Foundation, Kettering Fund, and Rotenberg Fund, which provided support. S.C. Nagamani is an awardee of the National Urea Cycle Disorders Foundation Research Fellowship and is supported by the Clinical Scientist Development Award from the Doris Duke Charitable Foundation.

\section{DISCLOSURE}

M Mokhtarani, D.F.C., and B.F.S. are employees of Hyperion. None of the other authors has a financial interest in Hyperion, although payments were made by Hyperion to each of the study sites for services provided in the conduct of the clinical studies on which these analyses were based, as well as to Accudata Solutions (D.A.M.) and M Marino for their assistance with the biostatistical analyses. A.S. is a paid consultant to Hyperion.

\section{REFERENCES}

1. Berry GT, Steiner RD. Long-term management of patients with urea cycle disorders. J Pediatr 2001;138(suppl 1):S56-60; discussion S60-51.

2. Lee B, Rhead W, Diaz GA, et al. Phase 2 comparison of a novel ammonia scavenging agent with sodium phenylbutyrate in patients with urea cycle disorders: safety, pharmacokinetics and ammonia control. Mol Genet Metab 2010;100:221-228.

3. Lichter-Konecki U, Diaz GA, Merritt JL 2nd, et al. Ammonia control in children with urea cycle disorders (UCDs); phase 2 comparison of sodium phenylbutyrate and glycerol phenylbutyrate. Mol Genet Metab 2011;103:323-329.

4. Smith W, Diaz GA, Lichter-Konecki $\mathrm{U}$, et al. Ammonia control in children ages 2 months through 5 years with urea cycle disorders: comparison of sodium phenylbutyrate and glycerol phenylbutyrate. J Pediatr 2013;162:1228-34, 1234.e1.

5. Diaz GA, Krivitzky LS, Mokhtarani M, et al. Ammonia control and neurocognitive outcome among urea cycle disorder patients treated with glycerol phenylbutyrate. Hepatology 2013;57:2171-2179.

6. Mokhtarani M, Diaz GA, Rhead W, et al. Elevated phenylacetic acid levels do not correlate with adverse events in patients with urea cycle disorders or hepatic encephalopathy and can be predicted based on the plasma PAA to PAGN ratio. Mol Genet Metab 2013;110:446-453.

7. Berry SA, Lichter-Konecki U, Diaz GA, et al. Glycerol phenylbutyrate treatment in children with urea cycle disorders: pooled analysis of short and long-term ammonia control and outcomes. Mol Genet Metab 2014;112:17-24.

8. Davison A, Hinkley D. Bootstrap Methods and Their Application. Cambridge University Press: London, 1997.

9. Kaplan E, Meier P. Nonparametric estimation from incomplete observations. J Am Statist Assoc 1958;53:457-481.

10. Peto R, Peto J. Asymptotically efficient rank invariant test procedures. J Royal Statist Soc Series A (General) 1972:185-207.

11. Breslow N. Tests of hypotheses in overdispersed Poisson regression and other quasi-likelihood models. J Am Statist Assoc 1990;85:565-571.

12. Dean CB, Nielsen JD. Generalized linear mixed models: a review and some extensions. Lifetime Data Analysis 2007;13:497-512.

13. Hardin JW. Generalized Estimating Equations (GEE). Wiley: Hoboken, NJ, 2005.

14. Maestri NE, McGowan KD, Brusilow SW. Plasma glutamine concentration: a guide in the management of urea cycle disorders. J Pediatr 1992;121:259-261.

15. Wilson CJ, Lee PJ, Leonard JV. Plasma glutamine and ammonia concentrations in ornithine carbamoyltransferase deficiency and citrullinaemia. J Inherit Metab Dis 2001;24:691-695.

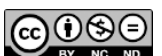

This work is licensed under a Creative Commons Attribution-NonCommercial-NoDerivs 3.0 Unported License. The images or other third party material in this article are included in the article's Creative Commons license, unless indicated otherwise in the credit line; if the material is not included under the Creative Commons license, users will need to obtain permission from the license holder to reproduce the material. To view a copy of this license, visit http://creativecommons.org/licenses/by-nc-nd/3.0/ 